\title{
An Ectopic, ACTH Producing, Oncocytic Carcinoid Tumor of the Thymus: Report of a Case
}

\author{
Izumi Yamaji, MD, Osamu Immura, MD, Takafumi Mito, MD, \\ Shigeo Yoshida, MD, Kazuaki Shimamoto, MD \\ and Takashi Minase*, MD
}

\begin{abstract}
A 39-year-old man with an ACTH producing oncocytic carcinoid of the thymus is reported here. His symptoms were pigmentation, facial and pretibial edema, and high blood pressure. Endocrinological examination revealed the ectopic ACTH syndrome and, especially, high content in the intrathoracic venous blood. On histological examination, the tumor was found to be composed of uniform eosinophilic cells, with no argentaffin granules being demonstrated. Ultrastructural findings revealed a large number of mitochondria and numerous distinct electron-dense neurosecretory granules in the cytoplasm. Abnormally high levels of ACTH, beta-endorphin and gamma-MSH were also found in this tumor tissue. By total extirpation of the tumor, clinical symptoms and laboratory data were entirely normalized.
\end{abstract}

Key Words: . Ectopic ACTH syndrome, Oncocytic carcinoid, Thymus

In 1962, Meader et al. ${ }^{1)}$ described 5 cases of Cushing's syndrome associated with nonendocrine tumors. Since then, many cases of the ectopic ACTH syndrome have been reported and it has also been found that these tumors originate most commonly in the lungs and, occasionally, in the pancreas or the thymus.

We had a patient with the ectopic ACTH syndrome originating in a tumor of the thymus diagnosed histologically as an oncocytic carcinoid.

Since a search of the literature failed to reveal any reports of similar cases, we present here our pre- and post-operative clinical findings on the case, along with histological and ultrastructural data, and discuss its clinical features and some considerations with regard to this form of tumor.

\section{CASE REPORT}

A 39-year-old man was admitted to our hospital on September 25, 1980, with general malaise, polydipsia, polyuria, pigmentation and edema of the face and legs.
In April, 1979, a diagnosis of glucosuria was made for the first time. Facial pigmentation and general malaise had begun in February, 1980, and he had experienced polydipsia, polyuria, and facial and pretibial edema from July, 1980.

Physical examination on admission disclosed a blood pressure of $180 / 100 \mathrm{mmHg}$ and a heart rate of 72 beats per minute. Brown pigmentation and edema were observed on the face. Moderate bilateral pretibial edema was also noticed. Neurologic examination showed no abnormality and the results of the remainder of the physical examination were unremarkable.

Laboratory studies revealed hyposthenuria, hypokalemia, alkalosis (Table 1), glucosuria and glucose intolerance (borderline type) with $50 \mathrm{~g}$ oGTT. Chest roentgenograms and computerized tomography showed a spherical mass at the antero-superior mediastinum.

During hospital admission but before surgery, blood pressure and urinary 17-OHCS and 17-KS remained at high levels (Fig. 1). Plasma ACTH and

From The Second Department of Internal Medicine, and *Department of Clinical Pathology,

Sapporo Medical College, Sapporo.

Received for publication July 28, 1983.

Reprint request to: Izumi Yamaji, MD, The Second Department of Internal Medicine, Sapporo Medical College, S-1, W-16, Chuo-ku, Sapporo 060, Japan. 
Table 1. Summary of main laboratory examinations, including hormonal determinations, in plasma, urine and tumor tissue. The numbers in parentheses indicate the normal value. Basal plasma levels of ACTH, beta-endorphin, cortisol and DOC clearly increased. GH, TSH, LH, FSH, PRL, aldosterone and androgen remained within normal range. Blood gas analysis and serum electrolytes represented hypokalemic alkalosis before the tumorectomy and these were normalized after surgery. In this tumor tissue, high contents of ACTH, beta-endorphin and gamma-MSH were found.

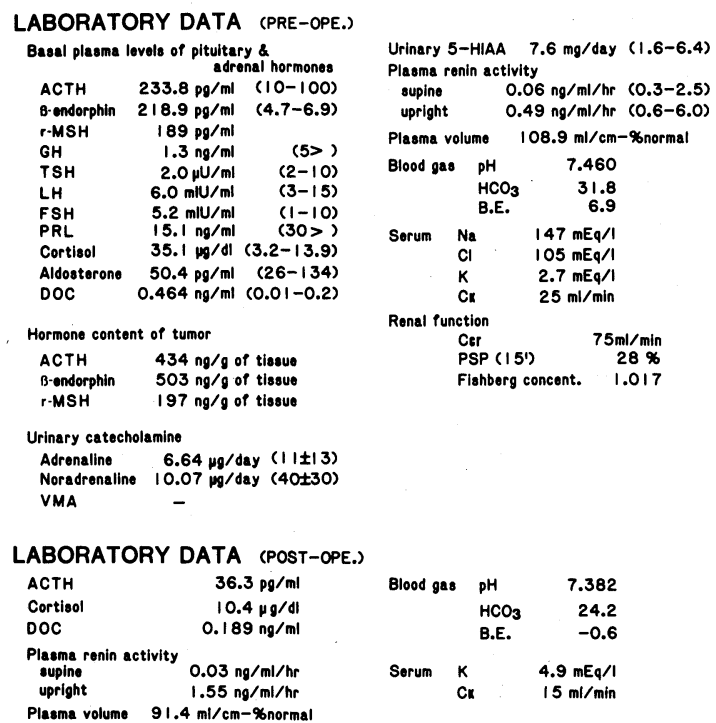

cortisol levels were elevated without any diurnal rhythm (Fig. 2). His plasma 11-deoxycorticosterone (DOC) level was also elevated. Other pituitary hormones (GH, TSH, LH, FSH and prolactin) in plasma, the plasma aldosterone concentration, the plasma androgen level, and the urinary excretion of catecholamines were within normal range (Table 1). He had little suppression of urinary 17 OHCS or 17-KS excretion following both $2 \mathrm{mg}$ and $8 \mathrm{mg}$ of dexamethasone, and no increase evoked by metyrapone (Fig. 3). Bilateral hypertrophy was demonstrated by adrenal scintigraphy with ${ }^{123}$ I-cholesterol. No abnormality was found on either cranial roentgenograms or computerized tomograms. Determination of ACTH in segmentally sampled venous blood revealed a marked

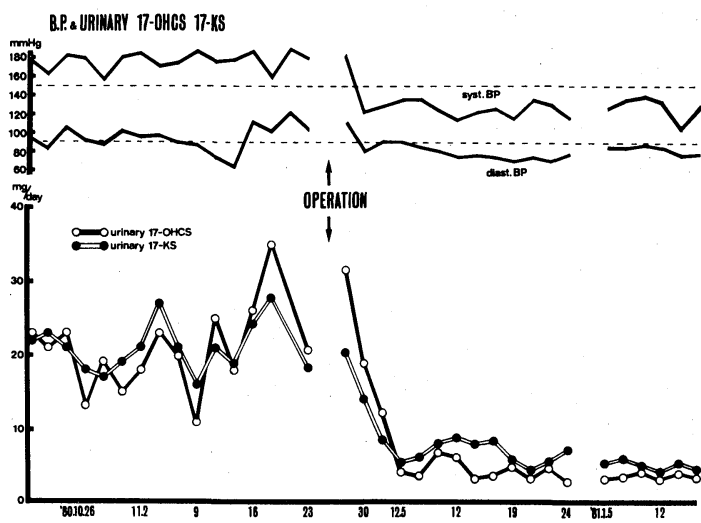

Fig. 1. Blood pressure, urinary 17-OHCS and 17-KS before and after the extirpation of the tumor. Blood pressure was kept within $160 \sim 180 / 80 \sim 120 \mathrm{mmHg}$ and the urinary excretion of 17-OHCS was $10 \sim 35 \mathrm{mg} /$ day before the tumorectomy. Both were entirely normalized after surgery.
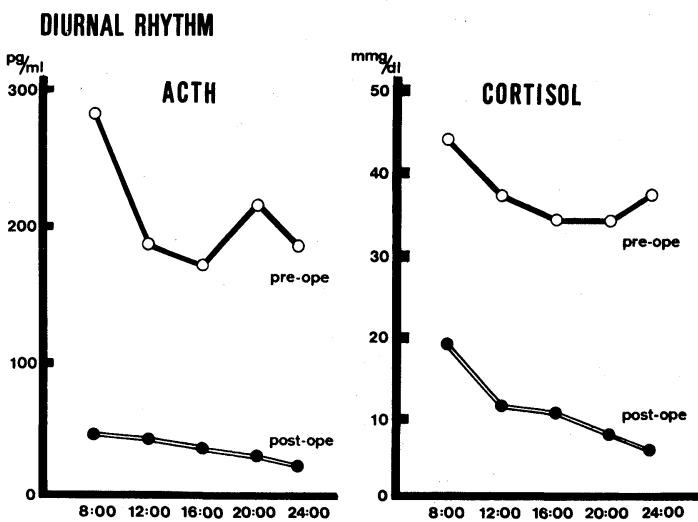

Fig. 2. Diunal rhythm of ACTH and cortisol. Preoperatively, the diurnal rhythm of ACTH and cortisol were of an abnormal pattern and abnormally high, but were normalized after surgery.

elevation of ACTH content in that sampled from the mediastinal region (Fig. 4). Thus, it was suggested that the ectopic ACTH production originated from the mediastinal tumor.

On November 25, 1980, a tumorectomy was performed. Grossly, the tumor was oval, smooth, gray-brown and partially hemorrhagic. After operation, blood pressure and urinary 17-OHCS and 17-KS rapidly normalized (Fig. 1), after which polydipsia, polyuria, hypokalemia and alkalosis 

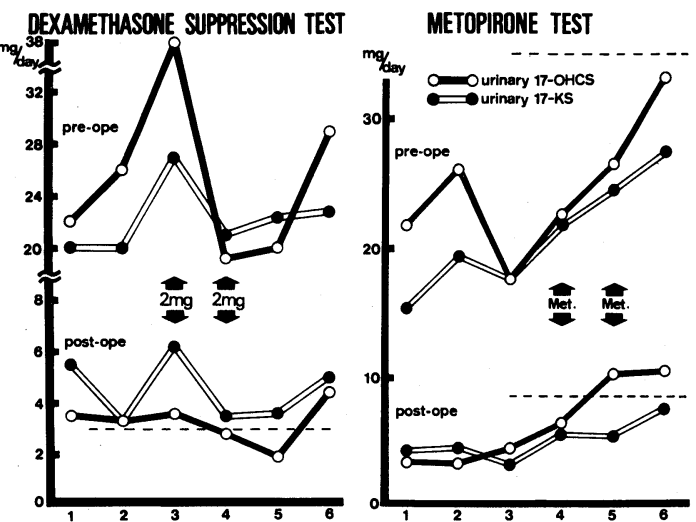

Fig. 3. Dexamethasone suppression and metyrapone (Metopirone) tests before and after the extirpation of the tumor. Preoperatively, there was hardly any response of urinary 17-OHCS to $2 \mathrm{mg}$ of dexamethasone and metyrapone, but after tumorectomy, normal response were observed on both tests.

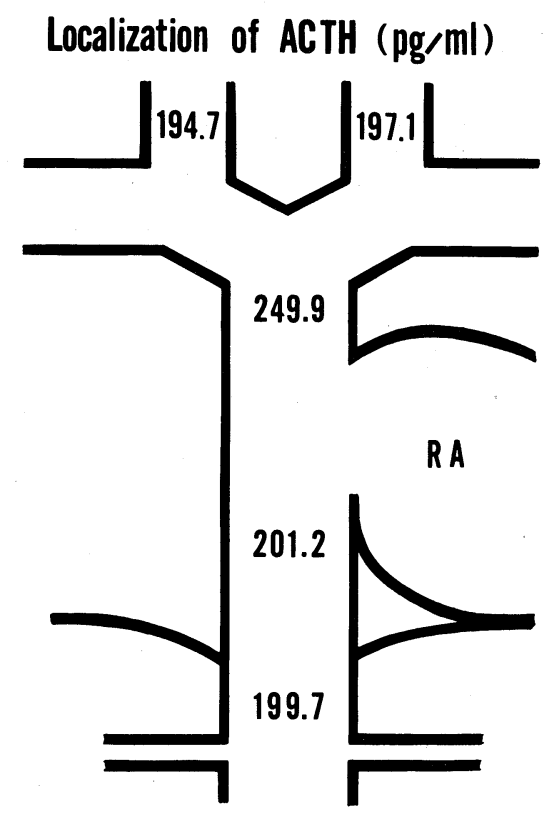

Fig. 4. The plasma concentration of ACTH in segmentally sampled venous blood. RA: right atrium. Plasma ACTH levels were elevated considerably as a whole, with the highest one being confirmed in the sample taken from the mediastinal region.

disappeared (Table 1). The diurnal rhythm of ACTH and cortisol (Fig. 2), dexamethasone suppression and metyrapone tests (Fig. 3) and

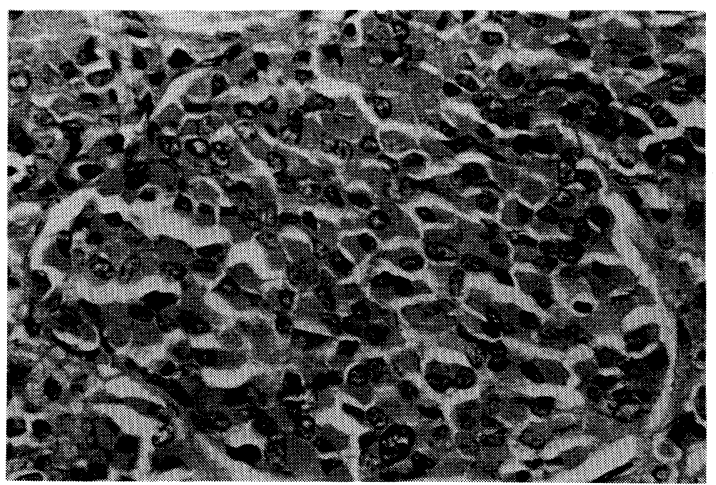

Fig. 5. Photometric microscopy (H-E stain) showed that the tumor was composed of relatively uniform eosinophilic cells.

$50 \mathrm{~g}$ o-GTT also returned to normal. Pigmentation and edema improved 2 months after the operation.

Photometric microscopy (Fig. 5) showed that the tumor was composed of relatively uniform eosinophilic cells and these cells were separated by vessel-rich fibrous tissues. Staining for argentaffin granules was negative. Rosette formation was not observed.

Electron microscopic examination (Fig. 6) revealed a large number of mitochondria and numerous distinct electron-dense neurosecretory granules within the cytoplasm. Tonofilaments and desmosomes were not identified.

From these findings, we concluded that the tumor was a very rare oncocytic carcinoid tumor of the thymus.

ACTH, beta-endorphin and gamma-MSH in the tumor tissues represented high levels: ACTH, 434 $\mathrm{ng} / \mathrm{g}$; beta-endorphin, $503 \mathrm{ng} / \mathrm{g}$; and gamma-MSH, $197 \mathrm{ng} / \mathrm{g}$ of tissue.

\section{DISCUSSION}

Hypercortisolism bringing about bilateral adrenocortical hyperfunction with a nonpituitary tumor was first described by Brown, W. H. ${ }^{2)}$ in 1928. In 1962, Meader et al. ${ }^{1)}$ reported 5 patients with nonendocrine tumors, bilateral adrenal hyperplasia, and hypercortisolism, and that their clinical signs were commonly hypokalemic alkalosis and pigmentation rather than the typical signs of Cushing's syndrome, i.e. central obesity, red striae. Liddle et al. ${ }^{3)}$ reported 13 cases of Cushing's 


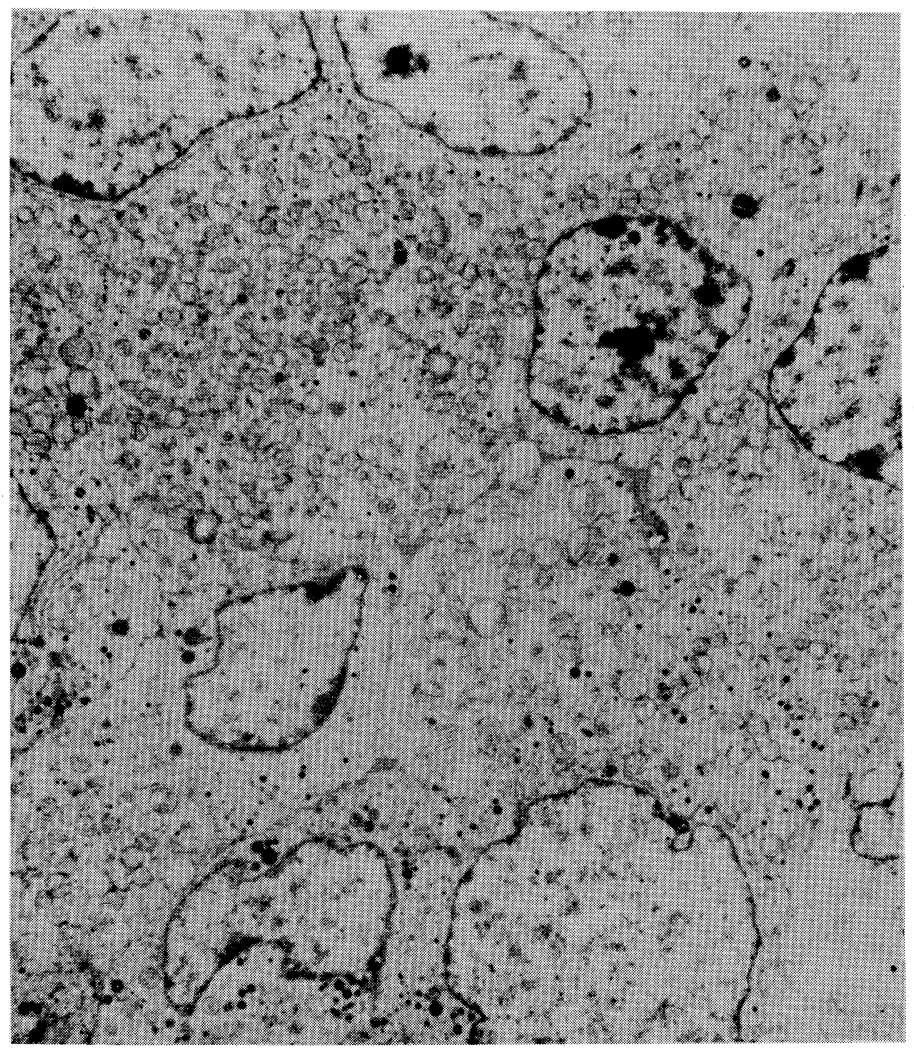

Fig. 6. Electron microscopic examination revealed a large number of mitochondria and numerous distinct electron-dense neurosecretory granules within the cytoplasm.

syndrome associated with nonpituitary tumors in 1963. Since then, this syndrome has generally been referred to as "the ectopic ACTH syndrome".

Many cases of the ectopic ACTH syndrome have been reported, with such tumors originating mainly in the lung, and occasionally in the gastrointestinal tract, pancreas or thymus. In our case, the clinical signs and symptoms were not central obesity and red striae, but hypokalemic alkalosis, pigmentation, hypertension and edema. A chest roentgenogram showed a mediastinal tumor without chest pain or any sign of the superior vena cava syndrome. Ectopic ACTH production was strongly suggested by some endocrinological examination findings. A diagnosis of an ectopic ACTH producing tumor was finally made after the measurement of plasma ACTH levels in the intrathoracic regions. Thus, it was considered, in our case at least, that the ACTH levels obtained from segmental or local venous sampling would be most useful in diagnosis of ectopic ACTH producing tumors.

A primary carcinoid tumor of the thymus was established as a specific entity distinct from thymoma by Rosai, J. and Higa, E. ${ }^{4)}$ in 1972. It is generally accepted that thymoma arise from thymic epithelial cells and that carcinoid tumors of the thymus arise from neural crest cells. About 100 cases of confirmed or probable carcinoid tumor of the thymus have been reported, ${ }^{5-7)}$ with some of these cases having the carcinoid syndrome $\mathrm{e}^{8-10)}$ or other endocrinological abnormalities.

Wick et al. ${ }^{10)}$ described microscopic and ultra- 
structural findings of the thymic carcinoid, and emphasized the necessity of electron microscopic observation in the diagnosis of thymic carcinoid tumors. According to their description, neurosecretory granules were not seen in thymoma but in carcinoid of the thymus, while desmosomes and tonofilaments were rare in carcinoid of the thymus but were frequent in thymoma. In addition, they suggested that some cases reported previously as thymoma with Cushing's syndrome were probably thymic carcinoid tumors.

Because the electron microscopic findings of our case revealed numerous neurosecretory granules and mitochondria-rich cytoplasm, this tumor was diagnosed as an oncocytic carcinoid tumor. The term oncocytoma was first used by Jaffe ${ }^{11}$ ) to describe tumors composed of cells containing numerous granular eosinophilic cytoplasm of the thymus. Several cases of oncocytomas have been reported in the salivary glands, ${ }^{11)}$ bronchial glands and duct, ${ }^{12,13)}$ and the lung. ${ }^{14)}$

In conclusion, although to our knowledge, no such case has ever been described before, based upon the above considerations we diagnosed this case as an ectopic ACTH producing oncocytic carcinoid tumor of the thymus.

ACKNOWLEDGMENT: The authors gratefully thank Kazuma Nakao and Hiroo Imura, Second Department of Internal Medicine, Kyoto University School of Medicine, for ACTH, beta-endorphin and gamma-MSH assays of the tumor tissue.

\section{REFERENCES}

1) Meador CK, Liddle GW, Island DP, et al: Cause of Cushing's syndrome in patients with tumors arising from "Nonendocrine" tissue. J Clin Endocrinol Metab 22: 693, 1962.

2) Brown WH: A case of pluriglandular syndrome. Lancet 2: 1022, 1928.

3) Liddle GW, Island DP, Ney RL, et al: Nonpituitary neoplasms and Cushing's syndrome. Arch Intern Med 111: 471, 1963.

4) Rosai J, Higa E: Mediastinal endocrine neoplasm, of probable thymic origin, related to carcinoid tumor. Cancer 29: 1061, 1972.

5) Manes JL, Taylor HB: Thymic carcinoid in familial multiple endocrine adenomatosis. Arch Path 95: 252, 1973.

6) Salyer WR, Salyer DC, Eggleston JC: Carcinoid tumors of the thymus. Cancer 37: 958, 1976.

7) Taguchi $Z$, Hasegawa $T$, Tomita $F$, et al: A case of primary thymic carcinoid. Jap J Thorac Surg 32: 137, 1979.

8) Nawata H, Maruyama T, Kato $K$, et al: A case of ACTH and beta-MSH producing malignant thymic carcinoid tumor and its relation to the ectopic ACTH producing malignant thymoma. J Jap Soc Intern Med 66: 1260, 1977.

9) Hata S, Kaneda N, Kunita H, et al: A case of ectopic ACTH syndrome induced by thymic carcinoid with remarkable fluctuation of pituitary-adrenal function. J Jap Soc Intern Med 66: 305, 1977.

10) Wick MR, Scott RE, Chin-Yang LI, et al: Carcinoid tumor of the thymus: a clinicopathologic report of seven cases with a review of the literature. Mayo Clin Proc 55: 246, 1980.

11) Jaffe RH: Adenolymphoma (oncocytoma) of the parotid gland. Am J Cancer 16: 1414, 1932.

12) Fechner RE, Bentinck BR: Ultrastructure of bronchial onkocy toma. Cancer 31: 1451, 1973.

13) Walter P, Warter A, Morand G: Carcinoide oncocytaire bronchique. Virchoes Arch A Path and Histol 379: 85, 1978.

14) Jeffrey LS, Andrew C, Klaus GB: Oncocytic carcinoid tumor of the lung. Am J Surg Pathol 4: 287, 1980. 